\title{
A Concise Review on Image Dehazing Techniques
}

\author{
Prince Owusu-Agyeman ${ }^{1 *}$, Wei Xie ${ }^{1}$, Yeboah Yao ${ }^{2}$ \\ ${ }^{1}$ School of Automation Science and Engineering, South China University of Technology, Guangzhou, China. \\ ${ }^{2}$ School of Automation, Guangdong University of Technology, Guangzhou, China. \\ * Corresponding author. Tel.: +13450461031; email: pagyeman@qq.com \\ Manuscript submitted December 11, 2018; accepted February 27, 2019. \\ doi: 10.17706/ijcee.2019.11.3.118-132
}

\begin{abstract}
Images acquired under the influence of bad weather conditions such as haze, fog and other aerosols are deteriorated due to the dispersal of atmospheric particles which lead to color fading and contrast reduction, making it challenging for human interpretation and object feature recognition. Several methods for image haze removal have been introduced over the recent years, which consist of approaches used to extrapolate information such as contrast, scene depth, color channels and so on. In this paper, we present a concise review of the current image dehazing methods. A comprehensive assessment and development on existing methods and related techniques is conducted based on their individual characteristics and principles. Qualitative and quantitative experimental evaluation of the state-of-the-art methods are conducted and discussed in depth. The paper further puts forward an overview of future trends within the research area.
\end{abstract}

Key words: Image dehazing, image enhancement, physical models, quality assessment.

\section{Introduction}

Outdoor images are frequently deteriorated by bad weather situation which includes fog, haze, smog and aerosol particles are suspended in the atmosphere causes the absorption and the scattering of the light which travels from the scene of interest to the camera, this is as a result of the atmospheric scattered light conjugating with the light acquired the camera which intends to have influence on the image contrast and color. Several methods of image haze removal in computer vision and image processing have been introduced over the recent years, which comprises of approaches used to withdraw information which includes contrast, scene depth, color channels and others. The aim of haze removal is to remove or lower hindrances caused by haze with dehazing approaches which intends to generate adequate visual properties and obtain profitable information. The model expressed below is mostly adopted $[29,37,39,40]$ to elaborate the haze formation in the areas of computer graphics and machine vision;

$$
\boldsymbol{I}(x)=\boldsymbol{J}(x) \boldsymbol{t}(x)+\boldsymbol{A}(l-t(x))
$$

From (1) $I$ represent the observed intensity of the haze image, $J$ indicates the scene radiance, $A$ indicates the global atmospheric light (Airlight) whiles $t$ indicates the transmission medium which identifies the rest of pixels which are not distorted and attains the camera. Here the main aim of dehazing is to recover $J$ from $I$ and $A$.

In this case, it is very essential and needful for computer vision systems and applications such as driver assistance systems [1], [2], outdoor video surveillance [3], [4], video assisted transportation [5], [6], remote 
sensing imagery [7], [8], and other related systems to enhance the visual properties and also emphasize on image features in order to recover essential information from corrupted images. Theoretically the approach of image haze removal which eliminates undesirable visual properties from image is referred to as image enhancement methods. On the contrary, image dehazing procedures varies from the conventional noise removal and contrast enhancement procedures since the existence of haze in an image relies on the distance of the camera and the scene of interest, which results in the distortion of colors in an image. The profound interest and the wide prospects of this research area have drawn the attention of developers in recent times. However, there are a wide range of image dehazing methods that are been introduced the research is still scattered and a complete theoretical system has still not been established. In particular, there is a lack of systematic summary of the advances in related work until now [9], [10] it is therefore prime and proper to conduct a survey on the advancement of image dehazing methods been proposed in recent years.

In this paper, a comprehensive assessment and development on existing methods of image dehazing and related techniques are been conducted based on their individual characteristics and principles respectively. The remainder of this paper is as follows; Section 2 elaborates the features and the principles of classified image dehazing methods. Section 3 discusses Qualitative Experimental Evaluation; Section 4 discusses Quantitative Experimental Evaluation. We finally draw conclusions on discussed dehazing algorithms overviews and the scope of future research tendencies in Section 5.

\section{Classifications of Dehazing Algorithms}

The classifications of image dehazing algorithms are based on various fundamentals and features, these procedures can be classified into three parts namely; Enhancement-based Technique and Restoration-based Approach, Fusion-based Approach. These above classified techniques or approaches can be also applied in video dehazing domain and are discussed in following subsections.

\subsection{Enhancement-Based Techniques}

Generally, the adaptation of image enhancement procedure is to eradicate the presence of noise and improve on the information acquired concurrently to provide high quality image, considering this form of approach. Histogram equalization is frequently adopted procedure on hazy images. This procedure does not seek to compensate the physical model of image degradation but instead enhances the contrast of an image by manipulating the gray scale values across a specific spectrum evenly distributed. Histogram equalization can be categorized into two parts namely the Local Histogram Equalization (LHE) and Global Histogram Equalization (GHE). GHE uses the information of the entire intensity values in an image for its transformation function which is method is suitable for global enhancement [11], aiming to redistribute the intensity of an image evenly the entire range of gray-levels which the cumulative histogram of the image is linear which is precisely effective for enhancing low contrast detail of an image [12]. GHE has the merits of low computations associated with high efficiency on various software platforms [13]. On the contrary, LHE allows the expansion to all local areas of the image that improves the local details based on local operations on low contrast images. A local histogram equalization method have been proven to provide better performance than global methods and establishes more local image details with higher image enhancement performance [14]. Xu et al. [15] proposed a comprehensive equalization model that merges contrast enhancement and white balancing into a unified framework for convex programming of the image histogram. Histogram equalization and a wavelet transform method are combined to enhance images, which can improve the gray distribution of images [16]. Xu et al. [17] have established a contrast limited adaptive histogram equalization (CLAHE) method to eliminate the effects of haze, which can reduce noise while enhancing the image contrast simultaneously. In [18] integrated the CLAHE procedure with the 
Weiner filter and [19] combined the CLAHE method with the finite impulse response filter to enhance the contrast of images. Retinex based algorithm was proposed by Land and McCann which is also known as retinal cerebral cortex theory on the basis of color perception by the human eyes [20]. Jobson et al. [21] proposed SSR algorithm on the basis of the center/surrounding Retinex method. The significance of this approach is to acquire the reflectance of an image by removing the ambient light. On the other hand, in way to retain a desirable balance between the dynamic range compression and the color constancy, Rahman et al. [22] proposed MSR algorithm which is the extending the SSR algorithm to multiple scales. However, the reflectance of an image has a bit reliance on the intensity of the illumination; the Retinex algorithm is compatible with image dehazing and can be formulated below:

$$
F(x, y)=R(x, y) L(x, y)
$$

where $F(x, y)$ denotes the acquired image, $R(x, y)$ and $L(x, y)$ represents the weights of reflectance and illumination respectively.

Zhang et al. [23] proposed an improved Retinex-based method to eliminate haze in a traffic video, the results proved that the proposed method can not only remove the haze but also enhance the vividness of the traffic video images. Fu et al. [24] proposed a variation framework for Retinex to process the reflectance and the illuminance from a single underwater image by decomposing, enhancing and combining after color correction, the strength of the Retinex algorithm are simple and suitable to implement. However, with this method the estimation of illuminance is based which requires a Gaussian convolution template that is unable to maintain edge information. In Frequency based enhancement algorithms hazy images that inherit low frequency properties are improved in the frequency domain, high-pass filters are introduce to image filtering in order to compensate low frequencies to improve high frequencies. This method converts an image into frequency domain by using Fourier transformation and other procedures, inverse transformation is being applied after the filtering operation is done in order to revert it back into spatial domain. Some of the main procedure of frequency based enhancement includes the wavelet transform [25], Curvelet transform [26] and homomorphic filtering [27]. The main goal of haze removal is to achieve visual clarity of an image for both human and machine recognition; the frequency based enhancement approach is quite useful. However, as it requires particular information in the process, the quality of the image is affected due to the absence of a degradation model and a physical model mechanism in the image enhancement processes which contributes to high computational complexity and the enhancement effects on color images tends to be unsatisfactory (Table 1).

Table 1. Comparative Analysis of Various Image Enhancement Techniques

\begin{tabular}{|c|c|c|c|c|}
\hline Subclasses & Methods & Merits & Demerit & Applications \\
\hline $\begin{array}{l}\text { Histogram } \\
\text { Equalization }\end{array}$ & $\begin{array}{l}\text { Global } \\
\text { histogram } \\
\text { Equalization }\end{array}$ & $\begin{array}{l}\text { Less computation. } \\
\text { High efficiency, eligible } \\
\text { for general enhancement } \\
\text { of both dark or bright } \\
\text { images }\end{array}$ & $\begin{array}{l}\text { Halo effect, color and } \\
\text { brightness } \\
\text { distortion, }\end{array}$ & $\begin{array}{l}\text { Comprehensive } \\
\text { images }\end{array}$ \\
\hline Retinex Theory & $\begin{array}{l}\text { Local histogram } \\
\text { Equalization }\end{array}$ & $\begin{array}{l}\text { High dynamic range } \\
\text { compression, adaptive color } \\
\text { constancy, Refined visual } \\
\text { image quality. }\end{array}$ & $\begin{array}{l}\text { High computational } \\
\text { complexity, } \\
\text { represents block } \\
\text { effects in an image } \\
\text { computational } \\
\text { complexity, presence } \\
\text { of halos effects in } \\
\text { refined image, } \\
\text { inability to preserve } \\
\text { edge properties }\end{array}$ & $\begin{array}{l}\text { Outdoor surveillance } \\
\text { systems }\end{array}$ \\
\hline
\end{tabular}


Single-scale retinex(SSR)

Less computations

Frequency- based enhancement

Curvelet transform

Wavelet

Transform

Homomorphic filtering
Contrast enhancement ability

Preserves edge properties

Multi-scale analysis and multi-resolution properties on image, Ability to resolve uneven light.
Imbalanced dynamic range compression and color constancy Blurry edges Inability to remove the cognitive content haze across the image over-darkness uneven illumination and over-brightness deficiency

Medical imaging, Face recognition applications
High computational complexity

\subsection{Restoration-Based Approach}

The restoration based procedure requires the analyzation of imaging mechanisms and the details of image degradation, by using inverse transformation the degraded images is refined into high quality image. Most researchers related to this field generalize this procedure as the physical model for image restoration based on image degradation. Most researchers have profoundly conducted comprehensive analysis on physical models and degradation mechanisms on the basis of Atmospheric scattering, which have achieved substantial developments in enhancing clarity of haze images. Atmospheric physics model proposed by McCartney [28] establishes that the model comprises of air light scattering model and light attenuation model. Some of the approaches evolve from this module includes interaction-based approach, partial differential based approach, single image dehazing approach based on prior knowledge and others. From the diagram Fig. 1 below illustrates the atmospheric scattering model, where the dotted lines represent the air light $(A)$ and the direct transmission $(t)$ respectively, finally the solid line represents the distance (d) between the camera and the observed scene.

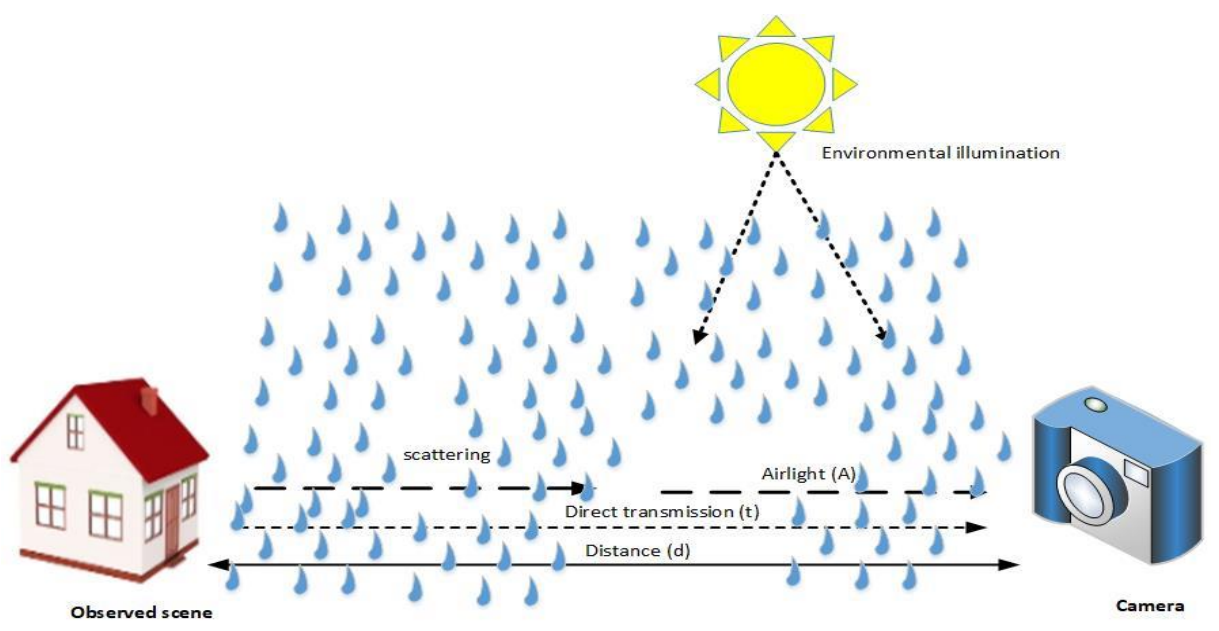

Fig. 1. Atmospheric scattering model.

Narasimhan's atmospheric model [29] derived from the previous model of McCartney's approach [28] which establishes that the entire radiant intensity captured by the camera is related to the linear superposition of the scene radiation light that includes scattered light penetrating the acquisition device .The attenuation of light intensity can be expressed as: 


$$
E_{d}(d, \lambda)=E_{0}(\lambda) e^{-\beta(\lambda) d}
$$

where $\lambda$ represents the wavelength of visible light, $d$ is the distance from the scene to the camera, $\beta(\lambda)$ denotes the atmospheric scattering coefficient whiles $E_{0}(\lambda)$ denotes the intensity of beam radiation at $\mathrm{x}=0$.

$$
E_{\alpha}(d, \lambda)=E_{\infty}(\lambda)\left(1-e^{-\beta(\lambda) d}\right)
$$

where $E_{\infty}(\lambda)$ represents the radiation beam intensity of the atmospheric light at infinity.

$$
\begin{gathered}
E(d, \lambda)=E_{d}(d, \lambda)+E_{\alpha}(d, \lambda) \\
E(d, \lambda)=E_{0}(\lambda) e^{-\beta(\lambda) d}+E_{\infty}(\lambda)\left(1-e^{-\beta(\lambda) d}\right)
\end{gathered}
$$

where the first part the equation describes the attenuated result of reflected light in the medium, and the second part represents the airlight that reflects the scattering of global atmospheric light. (6) can be simplified by letting $E(d, \lambda)=I(x)$ denotes haze image, $E_{0}(\lambda)=J(x)$ denotes scene radiance, $e^{-\beta(\lambda) d}=t(x)$ denotes transmission medium, represents Atmospheric airlight in (1).

\subsubsection{Interaction-based dehazing approach}

In [30] Narasimhan et al. introduced a single image interactive dehazing methods based on physical model which demands an individual to input the degraded part or sky region of an image based on bad weather, which unnaturally identifies minimum and maximum depth fields area to acquire approximate depth details. Images are been restored with the use of scene depth map estimation on the basis on atmospheric scattering model. In addition, it is obvious that such procedure is not complex since it does not demand accurate details of observed scene or weather condition, though its less complexity and efficiency compensates the contrast and the daytime colors of haze degraded images, this interactive based approach clearly enhances the contrast and the visual properties of haze images .However, due to the manipulative characteristics of method that requires the involvement of a user makes it less feasible in real time systems.

\subsubsection{Single image dehazing based additional information}

Tan et al. [31], [32] enhanced the algorithm proposed by Oakley el at which examined the degradation model on the basis of multi-parameter statistics under the perception that the depth of the scene is known and projected haze image restoration to color images. Robinson et al. [33] based on this analysis established a real time system based on atmospheric scattering model to expiate low contrast by eliminating the environmental light properties from each color channel. Generally, this procedure is emphasized assumption that the depth of the scene is recognized and that the results of the restored image is satisfactory. However, the method requires the availability of existing database to acquire correct scene depth details and hardware requirements which affects its capabilities in the real-time domain severely.

\subsubsection{Partial differential based approach}

The Partial differential approach is adopted by researchers in image dehazing which acquires the image gradient and depth of both local and global scene foremost then utilize the local minimum area to restore degraded image. Sun et al [34] adopted a method based on the assumption that the difference in scene depth which modifies atmospheric scattering model to a monochromatic model. Based on this enhanced model, images are divided into sky and non-sky regions, which are operated separately.

\subsubsection{Multi-image based dehazing approach}

Various approaches have been proposed in attempt to use depth information to estimate multiple scenes 
under different weather conditions and different polarizing filters. Under different weather conditions Narasimhan et al. [35] captures images of the same scene under two different weather conditions and examine the acquisition of depth information of the scene from different orientations, this method establishes geometric framework which is used for dehazing based on the influence atmospheric scattering on color. Different polarizing conditions is another procedure in multiple images dehazing, Schechner et al. [36] proposed a method that captures multiple images and by manipulating the direction of polarization direction of the polarizer which uses these acquired images to estimate the atmospheric optical polarization coefficient to recover colors and the contrast of the scene. Although the method achieves satisfactory results but it is difficult to be applicable in real-time systems due to the involvement of multiple scenes.

\subsubsection{Single image dehazing approach based on prior knowledge}

Remarkable progress has been made in recent years on single image dehazing which has been under-constrained challenge. Various approaches have been proposed based on prior knowledge or assumptions. Some of these corresponding algorithms are briefly discussed in the following:

Fattal et al. [37], proposed a method on basis of the assumption that the object surface shading and optical transmission are not correlated and established an independent component analysis (ICA) and a Markov random field (MRF) model to estimate the surface albedo, and recovered the image clarity from the haze image with medium transmission acquired from the scene.Fattal's approach applies physical features and produces impressive results. However, the algorithm has performance limitation for images with highly dense haze images, since the quality of the image depends on input image. Moreover, the method has a high computation which makes it relatively slow. Tan et al. [38] established a competent dehazing approach which is based on prior states. Firstly, it was assumed that the contrast of haze free images must be greater than that of the haze image and by the contrast maximization in degraded images due to haze influence produces impressive results with visual clarity. However, the dehazed images inherit irregular edge details, with applications like object and target recognition in which edge information is significant can be contradictive. Tarel et al. [39] proposed a dehazing approach which establishes image restoration through the basis of contrast enhancement. The algorithm inherits unique features like speed in processing over the previous approaches, though it is inconsistent and are influenced with factors like knowledge of viewers, evaluation, motivation and so on the approach has a limitation of preserving edge details. Thus the recovered image exhibits some haze on the edges of the image and also some color deformations. He et al. [40] proposed a dehazing algorithm known as the Dark Channel Prior (DCP) which is based on prior knowledge or assumption that for outdoor haze free images comprises of pixels with low intensities or approaching zero on at least one of the channels. This algorithm employs soft matting techniques to enhance image restoration and operatively supersedes the limitations of the previously discussed algorithms; the results of the restored images are impressive with visual clarity. However, the algorithm is computationally complex due to the construction of dark channel prior which makes the method slow and incapable in real time domain. An extensive progress has been achieved over the recent years motivated by Dark Channel Prior, Gibson et al. [41] employs median filter in the process of image restoration with DCP in order to make the method to have low computational complexity. However, the images restored inherit low brightness, less low structural similarity index measure (SSIM) and less peak signal-to-noise ratio (PSNR), Gao et al. [42] proposed a combination of DCP and existing a fast image dehazing approaches which uses negative correction to enhance the quality image restoration and also decrease the computational complexity of the algorithm. Dark Channel Prior motivated methods have been broadened to accommodate night-time images [43], [44], underwater images [45] and rainy or snowy conditions [46]. Lai et al. [47] propose to develop an optimal transmission map under a heuristic notion in the dehazing model where an 
objective function guarantees to have a global optimal solution, and the obtained transmission map accurately preserves the depth consistency of the same object. However, the method produces color distortions in the restored images. Zhu et al. [48] based on the machine learning approach of haze removal proposed an efficient and impressive color attenuation prior that form a linear model for scene depth of hazy images from a dataset, the method has a rapid processing and produces impressive results. However, it requires training of which the process is complex. See Table 2.

Table 2. Comparative Analysis of Various Image Restoration-Based Approaches

\begin{tabular}{|c|c|c|c|c|}
\hline Subclasses & Methods & Merits & Demerit & Applications \\
\hline \multirow[t]{8}{*}{$\begin{array}{l}\text { Restoration-Based } \\
\text { Approach }\end{array}$} & $\begin{array}{l}\text { Interaction-Based } \\
\text { Dehazing } \\
\text { Approach }\end{array}$ & $\begin{array}{lr}\text { Less } & \text { computation } \\
\text { complexity, } & \text { Visual image } \\
\text { clarity, } & \text { Contrast } \\
\text { enhancement } & \end{array}$ & $\begin{array}{l}\text { Multiple input image } \\
\text { requirement }\end{array}$ & $\begin{array}{l}\text { Comprehensive } \\
\text { images dehazing and } \\
\text { denoising }\end{array}$ \\
\hline & $\begin{array}{l}\text { Single Image } \\
\text { Dehazing based } \\
\text { Additional } \\
\text { Information }\end{array}$ & good recovery results & $\begin{array}{l}\text { Unavailability } \\
\text { existing database. } \\
\text { Hardware } \\
\text { requirements }\end{array}$ & Images dehazing \\
\hline & $\begin{array}{l}\text { Partial } \\
\text { differential based } \\
\text { approach }\end{array}$ & $\begin{array}{l}\text { Contrast enhancement, } \\
\text { Visual image clarity }\end{array}$ & $\begin{array}{l}\text { User interaction } \\
\text { requirement }\end{array}$ & Images dehazing \\
\hline & $\begin{array}{l}\text { Multi-image } \\
\text { Based Dehazing } \\
\text { Methods }\end{array}$ & good recovery results & $\begin{array}{l}\text { Multiple input image } \\
\text { requirement, less } \\
\text { capability in real time } \\
\text { domain }\end{array}$ & $\begin{array}{l}\text { Comprehensive } \\
\text { image dehazing and } \\
\text { denoising }\end{array}$ \\
\hline & Fattal et al. [37] & good recovery results & $\begin{array}{l}\text { Inability to restore } \\
\text { images with heavy } \\
\text { haze }\end{array}$ & Medical Image \\
\hline & Tan et al. [38] & Contrast enhancement & $\begin{array}{l}\text { Color oversaturation, } \\
\text { halo effects }\end{array}$ & $\begin{array}{l}\text { Gray Scale or Single } \\
\text { Color Image }\end{array}$ \\
\hline & Tarel et al. [39] & $\begin{array}{l}\text { Fast, Less computations, } \\
\text { Visual image clarity, contrast } \\
\text { enhancement }\end{array}$ & $\begin{array}{l}\text { Halo effects, color } \\
\text { distortions. Inability } \\
\text { to preserve edge } \\
\text { informati }\end{array}$ & $\begin{array}{l}\text { Traffic Surveillance } \\
\text { Systems }\end{array}$ \\
\hline & He et al. [40] & $\begin{array}{l}\text { High fidelity recovery } \\
\text { results, }\end{array}$ & $\begin{array}{l}\text { high computations } \\
\text { requirement, slow }\end{array}$ & $\begin{array}{l}\text { Single } \\
\text { Dehazing }\end{array}$ \\
\hline
\end{tabular}

\section{Qualitative Experimental Evaluation}

This section discusses the evaluation and the verification of various dehazing techniques. In image dehazing, it is mostly difficult to evaluate the performance and efficiency simply by human vision. For this reason, it is essential to compile all the characters of the various dehazing algorithms and analyze them in general, to ensure impartiality all the corresponding algorithms are realized on the same matlab platform. Qualitative visual Assessment is a subjective approach based on the visual qualities of dehazed images by various dehazing techniques. We discuss and emphasize on the visual qualities between the corresponding classifications of various image dehazing techniques which is based on the visual opinion of the processed image. We demonstrates the comparisons between various image dehazing algorithms cited in the previous section based on their visual qualities and performance on different haze images, From Fig. 2 below.(a) denotes the original hazy image,(b) denotes the output image of [49], (c) denotes the output image of [47], (d)denotes the output image of [40], (e) denotes the output image of [37], (f) denotes the output image of [39] and (g) denotes the output image of [48] methods. The results obtained from this haze images and their corresponding dehazing methods shows that (g) [48] appears to produce astonish results in terms of visual clarity and color fidelity in the process of image restoration processes amongst the presented 
dehazing algorithms whiles (b) [49] appears to inherit some gloomy effects on the processed image that intends to compromise the visual clarity of the restored image, therefore as a result makes the algorithm to produce images with low visual clarity and vividness.

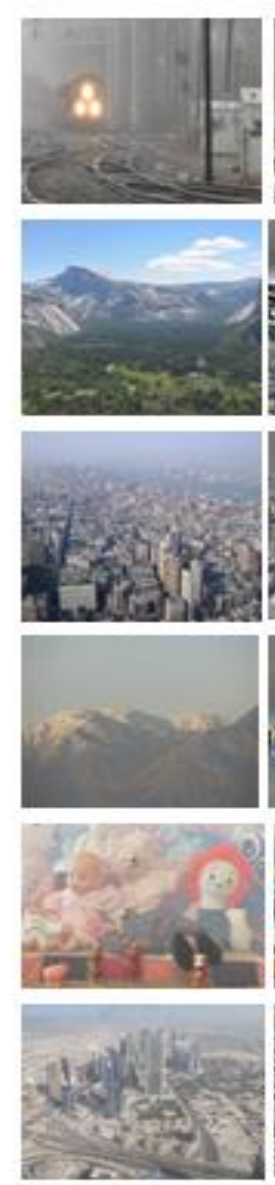

(a)
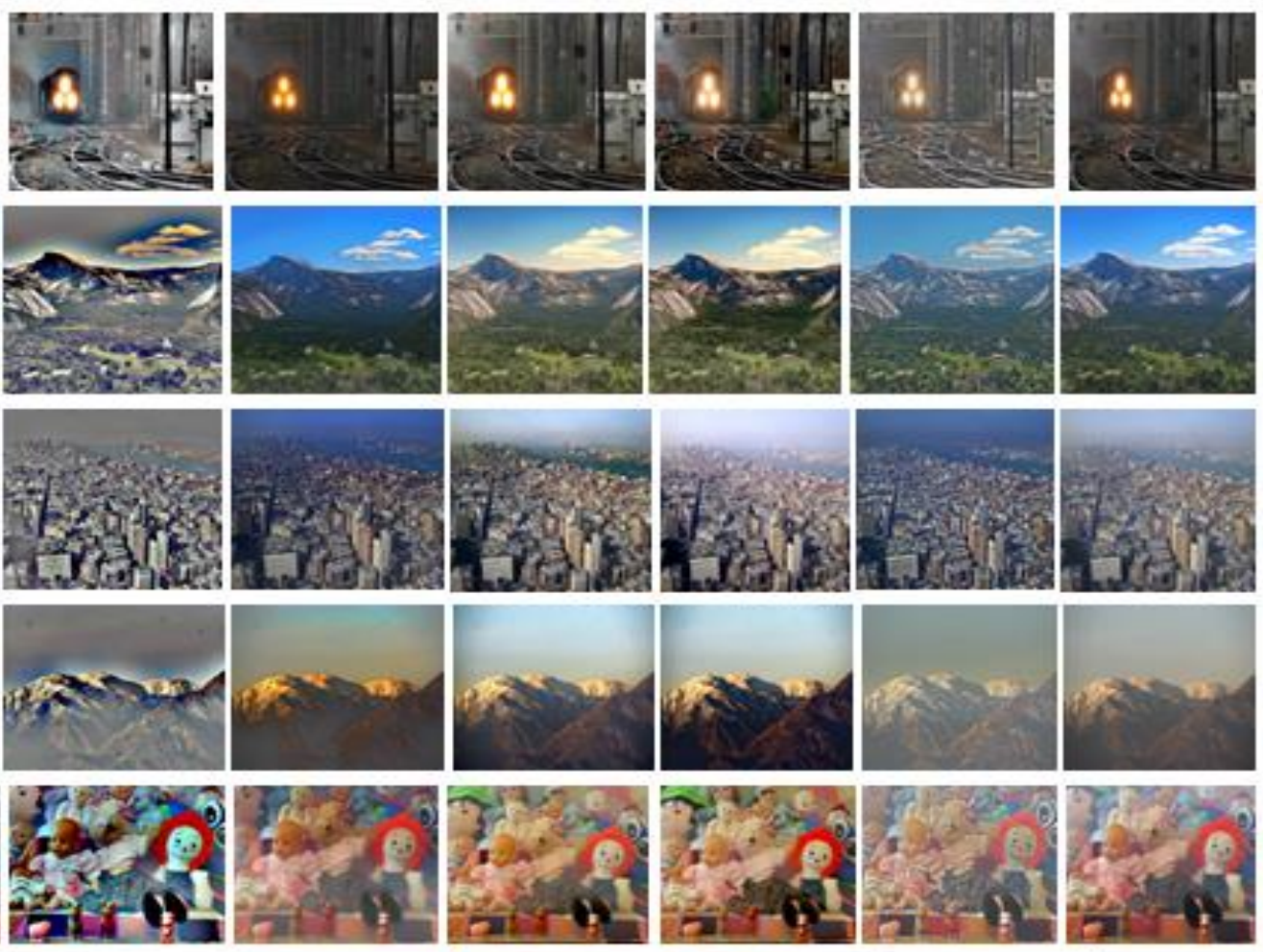

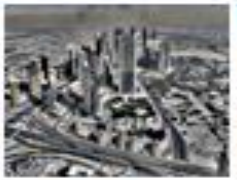

(b)

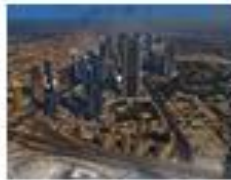

(c)

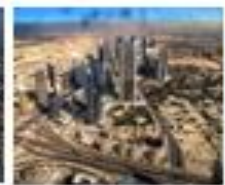

(d)

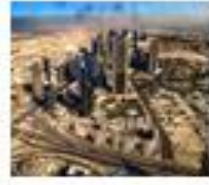

(e)

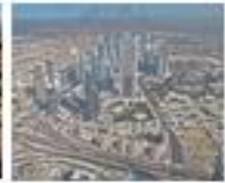

(f)

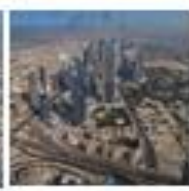

(g)

Fig. 2. Visual comparisons of various dehazing algorithms.

However, this criteria of assessment is simple and also can highlight on the visual quality of restored image, it is inconsistent and are influenced with factors like knowledge of viewers, evaluation, motivation and so on.

\section{Quantitative Experimental Evaluation}

In this section of this paper, we discuss the objective evaluation procedure which is used to assess quantitative data based on their corresponding objective metrics, on the contrary to the previous section above that highlights the visual clarity and comparisons of various dehazing algorithms based on visual opinion of a user subjectively is not an adequate assessment to be concluded on, since image dehazing algorithms are not constrained to only image restoration but also very potential in the improvement of the intrinsic properties of the restored image. For this reason, in order to determine the performances of intrinsic properties of the output images of various algorithms, the intrinsic metrics used to compare and evaluate quantitative data comprises Mean Squared Error (MSE), Peak Signal to Noise Ratio (PSNR), Signal to Noise Ratio (SNR) and Structural Similarity Index Measure (SSIM) respectively. However, the cognitive challenge of objective evaluation or quantitative assessment is the unavailability of reference image, since the computation of previously stated metrics demands the both the original image and reference image 
before the corruption of haze. The tables below represent the objective metrics performances and comparisons of the selected dehazing algorithms.

Mean squared error (MSE): The logical and consistently used intrinsic metric that can be estimated by calculating the average the squared intensity values of the distorted and reference image pixels [50]. It is expressed as:

$$
M S E=\frac{1}{M \times N} \sum_{i=1}^{M} \sum_{j=1}^{N}\left[f(i, j)-f^{\prime}(i, j)\right]
$$

where $M$ and $N$ represents the width and the height of the image respectively, $f(i, j)$ is the original image and $f^{\prime}(i, j)$ denotes the dehazed image.

Table 3. Comparative Results of MSE on Various Dehazing Algorithms

\begin{tabular}{lcccccc}
\hline \hline Image & Ling et al. [49] & Lai et al. [47] & He et al. [40] & Fattal et al. [37] & Tare et al. [39] & Zhu et al. [48] \\
\hline y1 & 4969.6 & 2934.7 & 4575.2 & 2968.7 & 1460.2 & 1480.1 \\
train & 1884.4 & 6104.7 & 10012.05 & 4581.88 & 1666.42 & 4260.24 \\
toys & 5604.8 & 3445.72 & 9851.17 & 1981.65 & 1330.09 & 1678.32 \\
snow & 2363.03 & 1931.26 & 12525.02 & 2225.83 & 489.96 & 549.11 \\
ny17 & 2687.3 & 3803.04 & 5853.63 & 4891.53 & 2918.25 & 327.96 \\
ny12 & 5614.2 & 2621.54 & 3320.56 & 3165.68 & 1651.578 & 744.12 \\
mountain & 2304.1 & 2431.9 & 4931.32 & 2205.28 & 6302.57 & 477.76 \\
hongkong & 1855.07 & 2332.04 & 5717.26 & 2193.34 & 3629.96 & 1600.59 \\
herzeliya & 2921.84 & 2280.7 & 2921.84 & 2474.05 & 1579.52 & 1135.64 \\
Dubai & 3783.47 & 6782.4 & 10099.02 & 2830.34 & 817.29 & 3164.42 \\
\hline \hline
\end{tabular}

Table 4. Comparative Results of PSNR on Various Dehazing Algorithms

\begin{tabular}{lcccccc}
\hline \hline Image & Ling et al. [49] & Lai et al. [47] & He et al. [40] & Fattal et al. [37] & Tare et al. [39] & Zhu et al. [48] \\
\hline y1 & 11.16 & 13.4 & 11.52 & 13.405 & 16.48 & 16.42 \\
train & 15.3 & 10.2 & 8.12 & 15.16 & 15.91 & 11.83 \\
toys & 10.64 & 12.75 & 8.19 & 15.16 & 16.89 & 15.88 \\
snow & 14.39 & 15.27 & 7.15 & 14.65 & 21.2 & 20.73 \\
ny17 & 13.8 & 12.32 & 10.45 & 11.23 & 13.47 & 22.97 \\
ny12 & 10.63 & 13.9 & 12.91 & 13.12 & 15.95 & 19.41 \\
mountain & 14.5 & 14.2 & 11.2 & 14.69 & 10.13 & 21.33 \\
hongkong & 15.44 & 14.45 & 10.55 & 14.71 & 12.53 & 16.08 \\
herzeliya & 14.23 & 14.55 & 13.47 & 14.19 & 16.14 & 17.57 \\
Dubai & 12.3 & 9.81 & 8.08 & 13.61 & 19 & 13.12 \\
\hline \hline
\end{tabular}

Peak signal to noise ratio (PSNR): The PSNR represents the index of the signal distortion. A large PSNR relates to a smaller image distortion [51]. It can be formulated as:

$$
P S N R=101 g \frac{f_{\max }^{2}}{M S E}
$$

where $f_{\text {max }}$ is the largest gray value, in general $f_{\text {max }}=255$.

Signal-to-noise ratio can be defined as the ratio of the average signal value to the standard deviation of the background which is formulated as: 


$$
S N R=\frac{\mu_{s i g}}{\sigma_{s i g}}
$$

where $\mu_{\text {sig }}$ represents average signal valued and $\sigma_{\text {sig }}$ represents standard deviation of the background respectively [52].

Table 5. Comparative Results of SNR on Various Dehazing Algorithms

\begin{tabular}{lcccccc}
\hline \hline Image & Ling et al. [49] & Lai et al. [47] & He et al. [40] & Fattal et al. [37] & Tare et al. [39] & Zhu et al. [48] \\
\hline y1 & 6.7 & 8.99 & 7.06 & 8.93 & 12.02 & 11.96 \\
train & 9.8 & 4.75 & 2.61 & 6 & 10.39 & 6.32 \\
toys & 7.01 & 9.125 & 4.56 & 11.52 & 13.25 & 12.24 \\
snow & 9.6 & 10.51 & 2.39 & 9.81 & 13.25 & 15.97 \\
ny17 & 9.15 & 7.61 & 5.77 & 6.55 & 8.79 & 18.28 \\
ny12 & 6.3 & 9.62 & 8.59 & 8.8 & 11.62 & 15.09 \\
mountain & 8.65 & 8.42 & 5.35 & 8.84 & 4.28 & 15.49 \\
hongkong & 10.45 & 9.45 & 5.56 & 9.73 & 7.54 & 11.09 \\
herzeliya & 9.25 & 9.42 & 8.31 & 9.21 & 11.16 & 12.59 \\
Dubai & 8.47 & 5.88 & 4.21 & 9.73 & 15.13 & 9.25 \\
\hline \hline
\end{tabular}

Structural similarity (SSIM) emphasizes on the human visual perception which is extremely considered for obtaining structural information from a scene. Wang et al. [53] proposed an SSIM index method to measure the restored image quality from the perspective of image formation by using the three parts of luminance comparison $l(x, y)$, contrast comparison $c(x, y)$ and structural comparison $s(x, y)$. The three components are combined to produce the similarity measure. It can be expressed as:

$$
S(i, j)=F(l(x, y), c(x, y), s(x, y))
$$

Table 6. Comparative Results of SSIM on Various Dehazing Algorithms

\begin{tabular}{lcccccc}
\hline \hline Image & Ling et al. [49] & Lai et al. [47] & He et al. [40] & Fattal et al. [37] & Tare et al. [39] & Zhu et al. [48] \\
\hline y1 & 6.7 & 8.99 & 7.06 & 8.93 & 12.02 & 11.96 \\
train & 9.8 & 4.75 & 2.61 & 6.02 & 10.39 & 6.32 \\
toys & 7.01 & 9.125 & 4.56 & 11.52 & 13.25 & 12.24 \\
snow & 9.6 & 10.51 & 2.39 & 9.81 & 13.25 & 15.97 \\
ny17 & 9.15 & 7.61 & 5.77 & 6.55 & 8.79 & 18.28 \\
ny12 & 6.3 & 9.62 & 8.59 & 8.8 & 11.62 & 15.09 \\
mountain & 8.65 & 8.42 & 5.35 & 8.84 & 4.28 & 15.49 \\
hongkong & 10.45 & 9.45 & 5.56 & 9.73 & 7.54 & 11.09 \\
herzeliya & 9.25 & 9.42 & 8.31 & 9.21 & 11.16 & 12.59 \\
Dubai & 8.47 & 5.88 & 4.21 & 9.73 & 15.13 & 9.25 \\
\hline \hline
\end{tabular}

The quantitative results and the comparison from our experiments of the selected image dehazing algorithms clearly emphasizes on their superiority and the inferiority respectively. From the tables above we demonstrates efficient reconstruction capabilities and performances of the corresponding algorithms based on the presented objective metrics With a ten image dataset was employed for the experiment and evaluation of selected algorithms, Table 3 discusses the MSE of the corresponding images which demonstrates that averagely Zhu et al. [48] algorithm achieve superiority with the lowest MSE whiles He et al. achieves inferiority with high MSE rate, Table 4 and Table 5 discusses the PSNR and SNR metrics of corresponding algorithms respectively, which indicates that Zhu et al. inherits superiority over the selected algorithms and He et al. inherits inferiority based on the assessment of PSNR and SNR respectively, Finally 
Table 6 discusses SSIM of the corresponding algorithms which demonstrates that averagely that zhu et al.'s algorithm achieves superiority over the rest of them the algorithms and with Retinex algorithm that attains an inferior performance based on the assessment of SSIM. Table 7 below discusses the computational complexity and real-time feasibility of the corresponding algorithms, the comparisons results of the computational time demonstrates that Zhu et al. presents an impressive computational speed and efficiency that outperforms the other presented algorithms. However, the comparison results of He et al. present an inferior performance in terms of computational speed and efficiency hence making it less feasible in the real-time domain.

Table 7. Comparative Results of Time \s on Various Dehazing Algorithms

\begin{tabular}{lccccc}
\hline \hline Image & Lai et al. [47] & He et al. [40] & Fattal et al. [37] & Tare et al. [39] & Zhu et al. [48] \\
\hline y1 & 681.25 & 353.4 & 67.33 & 167.58 & 37.33 \\
train & 403.28 & 167.43 & 57.19 & 61.97 & 17.7 \\
toys & 317.97 & 119.05 & 77.39 & 36.08 & 13.03 \\
snow & 388.59 & 365.84 & 22.50 & 252.04 & 22.39 \\
ny17 & 145.72 & 382.36 & 101.21 & 552.62 & 31.06 \\
ny12 & 2287.7 & 636.39 & 103.04 & 165.28 & 19.45 \\
mountain & 115.88 & 138.81 & 86.91 & 42.01 & 12.45 \\
hongkong & 136.97 & 504.92 & 103.43 & 436.57 & 28.87 \\
herzeliya & 75.38 & 16.317 & 152.56 & 1259.94 & 63.14 \\
Dubai & 62.01 & 665 & 101.95 & 315.12 & 37.71 \\
\hline \hline
\end{tabular}

\section{Conclusion}

This section of the paper emphasizes on the reviews of various image dehazing approaches and their classifications, we explored in-depth literature and various research woks on this dehazing field .In this paper the haze elimination procedures are categorized into classifications namely; enhancement-based and the restoration-based procedures for haze removal ,this classifications of dehazing algorithms and their corresponding merits, demerits and area of applications have been discussed thoroughly in details in the previous sections respectively. Experimental verification and evaluations have been demonstrated based on the performances of the corresponding algorithms in both subjective and objective criteria of assessment, where firstly we compared the selected algorithms based on the visual quality of the restored images which is influenced by human visual opinion, these criteria of assessment is simple but it is inconsistent. For this reason we also presents comparison of the corresponding dehazing algorithms with an objective assessment criteria which emphasizes on a quantitative evaluation metrics that address performances and the efficiency of the selected algorithms respectively. In view of the assessment above indicates that the enhancement-based approach of image dehazing enhances the contrast information to compensate low contrast of a degraded images based on a subjective assessment of human visual opinion which results in an unpredicted color distortions in the restored image mostly in the availability of intricate depths in restored images whiles the restoration-based approach classification of dehazing is correlated with the image degradation mechanism eligible to haze removal with different depth information however this procedure is time consuming with high computational complexity. The restoration-based approach appears to present an astonishing result in terms of performance and efficiency as compared to the enhancement-based approach of image dehazing. In addition, image dehazing has been a hotspot of research in the areas of machine vision in recent years of which some the works proves an impressive results under particular conditions, still needs to be improves upon. In view of the previous discussions, 
future works must be directed to the adaptation of single image processing under different fields and also fast image segmentation algorithms in order to overcome challenging task of robustness, efficiency and comprehensiveness in the real-time domain.

\section{Acknowledgment}

The authors would like to express their appreciation to the 303 research lab of the School of Automation Science and Engineering, South China University of Technology. This work was supported by Guangdong Provincial Science and Technology Major Project (2018B010108001).

\section{References}

[1] McCall, J. C., \& Trivedi, M. M. (2006). Video-based lane estimation and tracking for driver assistance: Survey, system, and evaluation. IEEE Transactions on Intelligent Transportation Systems, 7(1).

[2] Negru, M., Nedevschi, S., \& Peter, R. I. (2015) .Exponential contrast restoration in fog conditions for driving assistance. IEEE Transactions on Intelligent Transportation Systems, 16(4), 2257-2268.

[3] Huang, S. C. (2011). An advanced motion detection algorithm with video quality analysis for video surveillance systems.IEEE Trans. Circuits Syst. Video Technol., 21(1), 1-14.

[4] Xie, B., Guo, F., \& Cai, Z. X. (2012). Universal strategy for surveillance video defogging. Optical Engineering, 51(10), 101703.

[5] Halmaoui, H., Cord, A., \& Hautiere, N. (2011). Contrast restoration of road images taken in foggy weather. Proceedings of the IEEE International Conference on Computer Vision Workshops (ICCV Workshops) (pp. 2057-2063).

[6] Shehata, M. S., Cai, J., Badawy, W. M., Burr, T. W., Pervez, M. S., Johannesson, R. J., \& Radmanesh, A. (2008). Video-based automatic incident detection for smart roads: The outdoor environmental challenges regarding false alarms. IEEE Transactions on Intelligent Transportation Systems, 9(2), 349-360.

[7] Tao, S. Y., Feng, H. J., Xu, Z. H., \& Li, Q. (2012). Image degradation and recovery based on multiple scattering in remote sensing and bad weather condition. Optics Express, 20(15), 16584-16595.

[8] Makarau, A., Richter, R., Muller, R., \& Reinartz, P. (2014). Haze detection and removal in remotely sensed multispectral imagery. IEEE Transactions on Geoscience and Remote Sensing, 52(9), 5895-5905.

[9] Cai, C. T., Zhang, Q. Y., \& Liang, Y. H. (2015). A survey of image dehazing approaches. Proceedings of the 27th Chinese Control and Decision Conference (pp. 3964-3969). Qingdao, China.

[10] Wang, W. C., \& Yuan, X. H. (2017). Recent advances in image dehazing. IEEE/CAA Journal of Automatica Sinica, 4(3), 410-436.

[11] Abdullah-Al-Wadud, M., Hasanul-Kabir, M., Ali-Akber-Dewan, M., \& Chae, O. (2007). A dynamic histogram equalization for image contrast enhancement. IEEE Transactions on Consumer Electronics, 53(2), 593-600.

[12] Hall, L. (1973). Almost uniform distributions for computer image enhancement. IEEE Transactions on Computers, 23(2), 207-208.

[13] Wang, Q., \& Ward, R. K. (2007). Fast image/video contrast enhancement based on weighted thresholded histogram equalization. IEEE Transactions on Consumer Electronics, 53(2), 757-764.

[14] Khan, M. F., Khan, E., \& Abbasi, Z. A. (2014). Segment dependent dynamic multi-histogram equalization for image contrast enhancement. Digital Signal Processing, 25, 198-223.

[15] Xu, H. T., Zhai, G. T., Wu, X. L., \& Yang, X. K. (2014). Generalized equalization model for image enhancement. IEEE Transactions on Multimedia, 16(1), 68-82.

[16] Wang, L. J., \& Zhu, R. (2013). Image defogging algorithm of single color image based on wavelet transform and histogram equalization. Appl. Math. Sci., 7(79), 3913-3921. 
[17] Xu, Z. Y., Liu, X. M., \& Ji, N. (2009). Fog removal from color images using contrast limited adaptive histogram equalization. Proceedings of the 2nd IEEE Int. Congress on Image and Signal Processing (pp. 1-5). Tianjin, China.

[18] Al-Sammaraie, M. F. (2015). Contrast enhancement of roads images with foggy scenes based on histogram equalization. Proceedings of the 10th Int. Conf. Computer Science \& Education (pp. 95-101). Cambridge, UK.

[19] Yadav, G., Maheshwari, S., \& Agarwal, A. (2014). Foggy image enhancement using contrast limited adaptive histogram equalization of digitally filtered image: Performance improvement. Proceedings of the 2014 Int. Conf. Advances in Computing, Communications and Informatics (pp. 2225-2231). New Delhi, India.

[20] Land, E. H., \& McCann, J. J. (1971). Lightness and Retinex theory. J. Opt.Soc. Am., 61(1), 1-11.

[21] Jobson, D. J., Rahman, Z. U., \& Woodell, G. A. (1997). Properties and performance of a center/surround retinex. IEEE Trans. Image Process, 6(3), 451-462.

[22] Rahman, Z. U., Jobson, D. J., \& Woodell, G. A. (1996). Multi-scale Retinex for color image enhancement. Proceedings of the IEEE Int. Conf. Image Processing: Vol. 3. (pp. 1003-1006). Lausanne, Switzerland.

[23] Zhang, K., Wu, C. C., Miao, J. X., \& Yi, L. Z. (2013). Research about using the retinex-based method to remove the fog from the road traffic video. Proceedings of the International Conference on Transportation Information and Safety (pp. 861-867).

[24] Fu, X. Y., Zhuang, P. X., Huang, Y., Liao, Y. H., Zhang, X. P., \& Ding, X. H. (2014). A retinex-based enhancing approach for single underwater image. Proceedings of the 2014 IEEE Int. Conf. Image Processing, Paris, France (pp. 4572-4576).

[25] Zhu, R., \& Wang, L. J. (2014). Improved wavelet transform algorithm for single image dehazing. Optik-Int. J. Light Electron Opt., 125(13), 3064-3066.

[26] Starck, J. L., Murtagh, F., Candes, E. J., \& Donoho, D. L. (2003). Gray and color image contrast enhancement by the curvelet transform. IEEE Trans. Image Process, 12(6), 706-717.

[27] Cai, W. T., Liu, Y. X., Li, M. C., Cheng, L., \& Zhang, C. X. (2011). A self-adaptive homomorphic filter method for removing thin cloud. Proceedings of the 19th Int. Conf. Geoinformatics (pp. 1-4). Shanghai, China.

[28] McCartney, E. J. (1976). Optics of the Atmosphere: Scattering by Molecules and Particles (pp. 1-42). New York, USA: John Wiley and Sons, Inc.

[29] Narasimhan, S. G., \& Nayar, S. K. (2002). Vision and the atmosphere. International Journal of Computer Vision, 48(3), 233-254.

[30] Narasimhan, S. G., \& Nayar, S. K. (2003). Interactive (de) weathering of an image using physical models Proceedings of the IEEE Workshop on Color and Photometric Methods in Computer Vision (pp. 1-8).

[31] Tan, K., \& Oakley, J. P. (2001). Physics-based approach to color image enhancement in poor visibility conditions. J. Opt. Soc. Am. A, 18(10), 2460-2467.

[32] Tan, K., \& Oakley, J. P. (2002). Enhancement of color images in poor visibility conditions. Proceedings of the 2000 Int. Conf. Image Processing (pp. 788-791). Vancouver, BC, Canada.

[33] Robinson, M. J., Armitage, D. W., \& Oakley, J. P. (2002). Seeing in the mist: Real time video enhancement. Sensor Review, 22(2), 157-161.

[34] Sun, Y. B., Xiao, L., \& Wei, Z. H. (2007). Method of defogging image of outdoor scenes based on PDE. Journal of System Simulation, 19(16), 3739-3769.

[35] Narasimhan, S. G., \& Nayar, S. K. (2003). Contrast restoration of weather degraded images. IEEE Transactions on Pattern Analysis and Machine Intelligence, 25(6), 713-724.

[36] Schechner, Y. Y., Narasimhan, S. G., \&Nayar, S. K. (2001). Instant dehazing of images using polarization. Proceedings of the 2001 IEEE Computer Society Conf. Computer Vision and Pattern Recognition (pp. 
325-332). Kauai, HI, USA.

[37] Fattal, R. (2008). Single image dehazing. ACM Trans. Graph. (TOG), 27(3).

[38] Tan, R. T. (2008). Visibility in bad weather from a single image. Proceedings of the 2008 IEEE Conf. Computer Vision and Pattern Recognition, Anchorage (pp. 1-8). USA.

[39] Tarel, J. P., \& Hautiere, N. (2009). Fast visibility restoration from a single color or gray level image. Proceedings of the IEEE 12th Int. Conf. Computer Vision (pp. 2201-2208). Kyoto, Japan.

[40] He, K. M., Sun, J., \& Tang, X. O. (2009). Single image haze removal using dark channel prior. Proceedings of the IEEE Conf. Computer Vision and Pattern Recognition (pp. 1956-1963). New York, USA.

[41] Gibson, K. B., \& Nguyen T. Q. (2011). On the effectiveness of the dark channel prior for single image dehazing by approximating with minimum volume ellipsoids. Proceedings of the IEEE Int. Conf. Acoustics, Speech and Single Processing (pp. 1253-1256). Prague, Czech Republic.

[42] Gao, Y. Y., Hu, H. M., Wang, S. H., \& Li, B. (2014). A fast image dehazing algorithm based on negative correction. Signal Process., 103, 380-398.

[43] Pei, S. C., \& Lee, T. Y. (2012). Nighttime haze removal using color transfer pre-processing and dark channel prior. Proceedings of the 19th IEEE Int. Conf.Image Processing, Orlando (pp. 957-960). FL, USA.

[44] Jiang, X. S., Yao, H. X., Zhang, S. P., Lu, X. S., \& Zeng, W. (2013). Night video enhancement using improved dark channel prior. Proceedings of the 20th IEEE Int. Conf. Image Processing (pp. 553-557). Melbourne, VIC, Australian.

[45] Drews, P., Nascimento, E., Moraes, F., Botelho, S., \& Campos, M. (2013). Transmission estimation in underwater single images. Proceedings of the Int. Conf. Computer Vision Workshops (pp. 825-830). Sydney, NSW, Australia.

[46] Kawarabuki, H., \& Onoguchi, K. (2014). Snowfall detection in a foggy scene. Proceedings of the 22nd IEEE Int. Conf. Pattern Recognition (pp. 877-882). Stockholm, Sweden.

[47] Lai, Y.-S. Chen, Y.-L., \& Hsu, C.-T. (2012). Single image dehazing with optimal transmission map. Proceedings of the 21st International Conference on Pattern Recognition (ICPR2012) (PP. 388-391).

[48] Zhu, Q., Mai, J., \& Shao, L. (2015). A fast single image haze removal algorithm using color attenuation prior. IEEE Transactions on Image Processing, 24(11), 3522-3533.

[49] Tang, L., Chen, S., Liu, W., \& Li, Y. (2011). Improved Retinex image enhancement algorithm, Procedia Environmental Sciences, $A, 208-212$.

[50] Tang, K. T., Yang, J. C., \& Wang, J. (2014). Investigating haze-relevant features in a learning framework for image dehazing. Proceedings of the IEEE Conf. Computer Vision and Pattern Recognition (pp. 2995-3002). Columbus, OH, Italy.

[51] Wang, Y. K., \& Fan, C. T. (2014). Single image defogging by multiscale depth fusion. IEEE Trans. Image Process, 23(11), 4826-4837.

[52] Mazzetta, J., Caudle, D., \& Wageneck, B. (2005). Digital camera imaging evaluation. Electro Optical Industries, 8.

[53] Wang, Z., Bovik, A. C., Sheikh, H. R., \& Simoncelli, E. P. (2004). Image quality assessment: From error visibility to structural similarity. IEEETrans. Image Process, 13(4), 600-612.

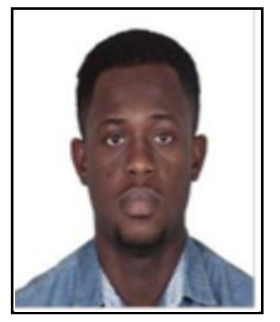

Prince Owusu-Agyeman received the B.Eng. degree in the fields of computer science and technology and M.Eng. electrical and computer engineering from the College of Automation Science and Engineering, South China University of Technology in 2013 and 2015, respectively. He joined the College of Automation Science and Engineering, South China University of Technology in 2015, as a $\mathrm{PhD}$. candidate. His current research interests include computer vision, image processing and real-time image enhancement. 


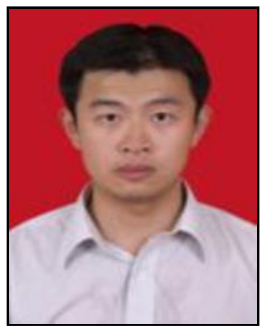

Wei Xie received the B. Eng. and M. Eng. degrees, both in Automation Department, from the Wuhan University of Science and Technology, China, in 1996 and 1999, respectively, and the Ph.D. degree from Kitami Institute of Technology, Japan, in 2003.

He worked as a postdoctoral researcher at the Satellite Venture Business Labortary, from 2003 to 2006. In 2006, he joined the College of Automation Science and Engineering, South China University of Technology, as an associate professor and was promoted to be a full professor in 2010. His research interests include control theory and application. He has published more than 50 papers in international journals and conferences. He is an inventor/co-inventor of 4 China patents. He is a member of the IEEE and has been a reviewer for several prestigious international journals (IEEE Transaction on Automatic control, IET Control Theory \& Applications, International Journal of Control and conferences (ACC, CCC, etc.). Prof. Xie's expertise areas are control theory and application, pattern recognition and intelligent system techniques.

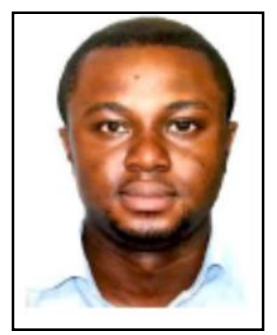

Yeboah Yao received the B.Eng. in the field of electronic information engineering from the Huazhong University of Science and Technology, Wuhan, China in 2011 and the M.Eng. from the South China University of Technology, Guangzhou, China in 2013 in the field of electrical and computer engineering. He subsequently received his Ph.D. in pattern recognition and intelligent systems in 2017 from South China University of Technology. Over the years, he has authored and co-authored several high-level publications sharing research contributions in machine learning, robotic vision and real-time visual perception. In 2017, he joined the School of Automation Science and Engineering, Guangdong University of Technology, as a postdoctoral research fellow. Dr. Yeboah's current research interests include deep learning techniques for autonomous robotics and pattern recognition for real-time applications. 